DOI: https://doi.org/10.33262/exploradordigital.v3i4.916

\title{
Plataformas virtuales y su impacto en la educación superior
}

Virtual platforms and their impact on high school

Pablo Velasteguí López. ${ }^{1}$, Sayda Cecilia Chamba Melo. ${ }^{2}$, Wilson Wilfrido Quille Chilborazo. ${ }^{3}$, Luis Efraín Velastegui López. ${ }^{4}$

\section{Resumen}

La educación en la actualidad es la encargada de transmitir conocimientos e información, además de proporcionar los medios, aptitudes y habilidades para producirlos y utilizarlos en la práctica pedagógica, creando nuevos escenarios donde se desarrolla el proceso educativo. Las aulas virtuales como espacio educativo se han convertido en un medio muy utilizado en las universidades, a través de ellas los docentes y estudiantes participan en un proceso de enseñanza aprendizaje mediante el empleo efectivo de las computadoras para acceder, comunicar, compartir y crear conocimientos que conducen al aprendizaje. La adecuación de tales prácticas tecnológicas en el ámbito educativo promueve la creación de nuevos entornos didácticos que afectan de manera directa tanto a los actores del proceso de enseñanza - aprendizaje como al escenario donde se lleva a cabo el mismo. La educación virtual es una nueva forma viable de enseñanza que viene a suplir necesidades, precariedades propias de la educación presencial y del mal uso de la tecnología educativa. El objetivo de la presente investigación es: establecer la percepción que tienen los docentes sobre el uso de las plataformas virtuales en la Universidad de Cotopaxi, extensión la Mana, donde se tomó un universo de 80 docentes de las distintas áreas, posteriormente se analizaron los datos para determinar las conclusiones.

Palabras claves: Tecnologías de la educación, aulas virtuales, proceso de enseñanza aprendizaje, tecnologías de la información y la comunicación.
Abstract
Education is currently responsible for transmitting knowledge and information, in addition to providing the means, skills and abilities to produce them and use them in pedagogical practice, creating new scenarios where the educational process develops. Virtual classrooms as an educational space have become a widely used medium in

\footnotetext{
Explorador Digital, Ambato, Ecuador, pablohomerovelastegui@hotmail.com schamba01@institutos.gob.ec wquille@institutos.gob.ec
}
2 Instituto Superior Pedagógico Intercultural Bilingüe Ab. Martha Bucarán, Ecuador,
3 Instituto Superior Pedagógico Intercultural Bilingüe Ab. Martha Bucarán, Ecuador,
${ }^{4}$ Ciencia digital, Ambato, Ecuador, luisefrainvelastegui@cienciadigital.org 
universities, through them teachers and students participate in a teaching-learning process through the effective use of computers to access, communicate, share and create knowledge that They lead to learning. The adaptation of such technological practices in the educational field promotes the creation of new didactic environments that directly affect both the actors of the teaching-learning process and the scenario where it is carried out. Virtual education is a new viable form of education that meets the needs, precariousness of face-to-face education and the misuse of educational technology.

The objective of the present investigation is: to establish the perception that teachers have about the use of virtual platforms at the University of Cotopaxi, extension Mana, where a universe of 80 teachers from different areas was taken, later the data were analyzed to determine the conclusions.

Keywords: Educational technologies, virtual classrooms, teaching - learning process, information and communication technologies.

\section{Introducción}

Las aulas virtuales en varias investigaciones teóricas realizadas por Abrigo, I. (2015), Chamba, L. (2015) y Delgado, M. (2010), afirman que el aula virtual es un espacio caracterizado por la innovación educativa, el énfasis en la actividad sobre los contenidos, la participación creativa de los alumnos y el aprendizaje colaborativo que se realiza mediante el uso de elementos tecnológicos.

La educación virtual es una nueva forma viable de enseñanza que viene a suplir necesidades, precariedades propias de la educación presencial, de saberes y de prácticas educativas mediante soportes virtuales, sin barreras de tiempo y distancia, que permite la construcción de un gran campus virtual a nivel planetario, sustentando en los mecanismos de la interactividad e interconectividad que se desprenden de la incorporación de las tecnologías en el campo educativo.

Las aulas virtuales en la educación según la investigación realizada por Porter, L. (1997), define que la evolución de las plataformas educativas se muestra muy ligada al desarrollo de la sociedad de la información y del conocimiento, y más en concreto, al de los sistemas educativos que tienden progresivamente a adaptarse a las necesidades reales del mundo laboral.

En este marco de innovación y cambio, la opción de generar entornos virtuales de aprendizaje basados en las tecnologías de la información y la comunicación, supone responder de forma integral los requerimientos que impone la sociedad del conocimiento, y a las nuevas necesidades del entorno educativo. Es dentro de este contexto de innovación donde surgen las plataformas educativas virtuales.

La educación actual afronta múltiples retos y uno de ellos es dar respuestas a los profundos cambios sociales, económicos y culturales que se prevén para la llamada "Sociedad de la Información y el Conocimiento" en esta era de la información y la comunicación, siendo el internet el que ha generado un enorme interés en todos los 
ámbitos de nuestra sociedad y gracias a su creciente uso con fines educativos se ha convertido en un campo abierto a la reflexión e investigación.

Sabiendo que las aulas virtuales son un medio en que los educadores y educando se encuentran para realizar actividades que conducen al aprendizaje, y que el concepto de conocimiento es la capacidad para transformar datos e informaciones en acciones efectivas y eficaces.

La modalidad de formación por medio de las aulas virtuales, ha mostrado mayor efectividad, despedazando las barreras de tiempo, al brindar técnicas, métodos y recursos que crean más efectivo y flexible el proceso de enseñanza - aprendizaje, ya que consienten el uso de las tecnologías de información y comunicación.

Las aulas virtuales brindan al estudiante y docente la oportunidad de obtener nuevos conocimientos mediante su manipulación a través del proceso de enseñanza aprendizaje, son medios que permiten desarrollar diferentes actividades académicas y de investigación encontrando en este entorno una comunidad propia para realizar, orientar nuevos conocimientos a través de la comunicación y distribución.

\section{Factores de importancia en la educación virtual}

Los factores que se consideran importantes según las investigaciones obtenidas de Bernad, E. y Camacho, M. (2016) y Machado, A. (2004) afirman que son los siguientes:

- Exploración: El uso de internet como una herramienta de exploración les abre al profesor y al estudiante universitario las puertas de una fuente inagotable de información y recursos.

- Experiencia: El estudiante virtual se ve involucrado en una nueva experiencia social y de aprendizaje que puede incluir comunicaciones directas con su profesor, discusiones con sus compañeros de curso o estudio individual de contenidos a su propio ritmo.

- Compromiso: Los recursos virtuales ofrecen una oportunidad única al estudiante de compartir experiencias con otros, lo que refuerza el sentido de colaboración y de comunidad. Además, el estudiante recibe el control de su tiempo y sus recursos, puede escoger el mejor camino de aprendizaje de acuerdo con sus preferencias y capacidades.

- Flexibilidad: Desde cualquier lugar y a cualquier hora, los estudiantes pueden tener acceso a sus cursos virtuales. Se estima que aproximadamente $80 \%$ de las empresas ya tienen solucionado su acceso a internet, por lo que los obstáculos de acceso de los estudiantes que trabajan ya están superados.

Las aulas virtuales son muy importantes en la educación, se encuentra todos los factores que existen en el aula, este entorno educativo, permite al estudiante realizar sus trabajos en línea de forma independiente, así como las oportunidades de interacción que normalmente existen en el aula y en la enseñanza cara a cara con el docente y posibilita el desarrollo del proceso de aprendizaje aplicando las tecnologías de la información y comunicación Tics.

Las aulas virtuales les permiten a los estudiantes e instructores comunicarse utilizando funciones como audio, video, chat de texto, pizarra interactiva y uso compartido de aplicaciones. El propósito del estudio adopta aulas virtuales sincrónicas y los apéndices 
especiales ofrecen información actualizada sobre grupo de noticias y listas de correos, sitios web de instrucción, recursos en línea para subvenciones y propuestas, y muchos más.

El modelo educativo en línea en varias fuentes bibliográficas realizadas de Domínguez, J. (2013), Khvilon, E. (2002) y Lepez, L. (2014), afirman que la educación virtual el aprendizaje está centrado en el alumno y su participación activa en la construcción de conocimientos le asegura un aprendizaje significativo. Con esto, se definen los contenidos y actividades para un curso partiendo de la estrategia didáctica diseñada por el docente. El estudiante universitario realiza su proceso de aprendizaje a partir de dichos contenidos y actividades, pero, sobre todo, a través de su propia motivación por aprender, de la interacción con otros compañeros y de la guía y asesoría de su docente.

El estudiante universitario mediante las plataformas virtuales aprende de forma más activa y dinámica ya que no sólo recibe la instrucción del docente, sino que aprende a través de la búsqueda de información, la autorreflexión, analizando cada concepto investigado y obteniendo nuevos conocimientos al momento de realizar las diversas actividades de manera individual en el proceso de la enseñanza - aprendizaje.

A través de estas herramientas tecnológicas la educación se ha convertido más fácil ya que el docente y estudiante pueden estar conectados mediante el internet, para realizar sus trabajos, evaluaciones, investigar, etc., sin duda permite enriquecer obteniendo nuevos conocimientos al manipular estas tecnologías virtuales.

\section{Educación Virtual}

La educación virtual en varias fuentes investigadas de Gallo, R. (2013), Gómez, L. (2012) y Moreno, R. (2014), definen que las nuevas tecnologías de la información y comunicación posibilitan la creación de un nuevo espacio social - virtual para las interrelaciones humanas, este nuevo entorno, se está desarrollando en el área de la educación, porque posibilita nuevos procesos de aprendizaje y transmisión del conocimiento a través de las redes modernas de comunicaciones.

El espacio virtual, que le llamo aulas sin paredes, cuyo mejor exponente actual es la red Internet, no es presencial sino representacional, no es próxima, sino distal, no es sincrónico, sino multicrónico, y no se basa en recintos espaciales con interior, frontera y exterior, sino que depende de redes electrónicas cuyos nodos de interacción pueden estar determinados por diversos países.

\section{Mundos virtuales aportan al espacio real}

Sus características son:

$\checkmark$ En la representación para comprender mejor lo real.

$\checkmark$ Son mundos en potencia, que nadie puede prever la magnitud de su avance.

$\checkmark$ Se entremezclan entre lo real y lo virtual, lo potencial y lo actual.

$\checkmark$ Se alimentan de la vida intermedia de los lenguajes simbólicos.

$\checkmark$ Se alimentan de la realidad visible.

$\checkmark$ Son intangibles, de lo sensible, la concepción y la percepción.

$\checkmark$ Son sistemas que procuran darnos la ilusión más convincente posible de inmersión funcional dentro de un mundo sintético. 
$\checkmark$ Representa una situación real, la tele virtualidad, la tele intervenciones o de tele robótica, también discutiblemente reunidas bajo la etiqueta de telepresencia.

$\checkmark$ Se esconde bajo la inconciencia de lo potencial humano.

$\checkmark$ Es parte del potencial aristotélico, aptitud para recibir una forma.

$\checkmark$ La potencia hace que encuentre vías de actualización, en virtudes necesarias para su determinación.

\section{Los entornos virtuales entes socializadores}

La comunicación educativa refuerza las enormes potencialidades comunicativas que ofrecen los entornos virtuales en cuanto a la socialización, lo paradójico es su centralidad favorece le des jerarquización de las relaciones humanas. Su ubicuidad fomenta la distribución, intercambio y circulación de información, ideas y conocimientos. Su plasticidad permite la aprehensión sensible de modelos conceptuales aparejando un enorme salto el modo de abordad los objetos de conocimiento.

\section{El conocimiento virtual}

$\checkmark$ Surge de la virtud del hombre en el alcance de conocer la verdad y la realidad de las cosas.

$\checkmark$ Es dependiente de las leyes de las redes virtuales, y la reacción personal a partir de la virtud humana.

$\checkmark$ Es dependiente de las leyes de las redes virtuales, y la reacción personal a partir de la virtud humana.

$\checkmark$ Está estructurado de acuerdo a los parámetros de versatilidad.

$\checkmark$ Facilidad de acceso y rapidez de descarga.

$\checkmark$ Tiene posibilidad de manipularlo interactuar con cualquier usuario remoto.

$\checkmark$ Se puede generarse un debate, sin tener un tema específico en Internet.

$\checkmark$ Se pude interactuar con individuos de cualquier parte del mundo.

$\checkmark$ No es Internet, sino el saber que existe en Internet.

\section{Educación y virtualidad}

La educación es un ente socializador que impulsa al conocimiento epistemológico a sumergirse, hasta llegar el proceso de dialecticidad. Sin embargo, como ya antecedemos, la virtualidad no tiene límites, ni reglas, es espontáneo y transformador que parte de la virtud humana.

En las sociedades se constituyen la diferenciación de las clases. Las clases sociales tiende a estar concentradas regionalmente, la división entre los barrios de las ciudades, sin embargo, el espacio virtual o la virtualización, en cambio aparece como un espacio homogéneo, sin diferencias de clases, razas o sexos, que integra a grandes masas sociales sin que tenga estas limitaciones a acceso a la educación.

La educación virtual enmarca la utilización de las nuevas tecnologías, hacia el desarrollo de metodologías alternativas para el aprendizaje de alumnos de poblaciones especiales que están limitadas por su ubicación geográfica, la calidad de docencia y el tiempo disponible. 
LA UNESCO define como entornos de aprendizajes que constituyen una forma totalmente nueva en relación con la tecnología educativa, un programa informático interactivo de carácter pedagógico que posee una capacidad de comunicación integrada. Son una innovación relativamente reciente y fruto de la convergencia de las tecnologías informáticas y de telecomunicaciones que se ha intensificado durante los últimos años. Por otro lado, la educación virtual como la educación del siglo XXI, tiene los siguientes principios.

$\checkmark$ La autoeducación

$\checkmark$ La autoformación

$\checkmark$ La desterritorialización

$\checkmark$ La descentración

$\checkmark$ La virtualización

$\checkmark$ La tecnologización

$\checkmark$ La sociabilidad virtual

La virtualidad no es algo nuevo en la historia de la humanidad. Hoy en día la tecnología nos brinda ese potencial, de posibilidad de incluso visionarlo con nuestros propios ojos, reconstruir la imaginación de hacer realidad visual nuestras ideas, se trata de lo que paradójicamente llamamos realidad virtual.

La educación virtual es una estrategia educativa basada en el uso intensivo de las herramientas tecnológicas, estructuras operativas flexibles y métodos pedagógicos, altamente eficientes en el proceso de enseñanza - aprendizaje, que permite que las condiciones de tiempo, espacio, ocupación o edad de los estudiantes no sean factores limitantes o condiciones para el aprendizaje.

\section{Educación virtual, aprendizaje autónomo y construcción de conocimiento}

La educación virtual superior en varias investigaciones realizadas de Delgado, M. (2010), Sierra, C. (2012), Vargas, R. (2004) y Zambrano, W. (2012), afirman que responden a la necesidad de una nueva visión y modelo de enseñanza - aprendizaje expresada en el marco de una sociedad en permanente cambio que exige replantear la manera de elaborar, adquirir y transmitir conocimiento con criterios de calidad y equidad. Esta clase de educación permite una formación sin limitaciones desde cualquier parte, a toda hora y por cualquier persona, lo que cobra cada vez más valor, sentido y significado en estos tiempos de globalización, información y conocimiento.

Esta modalidad denominada educación virtual se centra en el proceso de enseñanza aprendizaje apoyado por las TIC en una plataforma informática que facilita la interactividad entre alumnos, docentes, compañeros de estudio y materiales multimedia puestos a su alcance en la red Internet. También, constituye un proceso sistemático que implica establecer y definir con claridad los objetivos de aprendizaje, componentes, interrelaciónales, mecanismos de calidad, procedimientos, seguimiento y control de información. 
Sin duda, estos paradigmas de hoy exigen y obligan a nuevas y profundas transformaciones en las dinámicas de enseñanza - aprendizaje de las disciplinas y saberes en la educación superior. Estos nuevos retos imponen un enfoque socio constructivista y un modelo más flexible, móvil, pertinente y autónomo centrado más en aprender haciendo que en enseñar, basado en el uso creativo de herramientas y en recursos de formación a través de un aprendizaje colaborativo, significativo, distribuido y dinámico con formas innovadoras e interactivas de trabajo.

Estas nuevas dinámicas implican en un modelo donde los procesos de aprendizaje sean cada vez más eficientes y más efectivos con el fin de contribuir a la construcción y la divulgación de conocimiento no sólo en su esencia, sino también en su aplicación. Por lo tanto, necesita vislumbrar las tendencias, amenazas, cambios y oportunidades que enfrentan los modelos de aprendizaje clásicos que han sido soportados históricamente en un enfoque basado en el acopio y transmisión fragmentada y memorística de información, frente a una nueva formación más amplia, diversa y flexible que constantemente es retada por la rápida incorporación de nuevas experiencias pedagógicas, didácticas y tecnológicas.

Muchos aspectos de la humanidad que tienen que ver con su propia evolución van cambiando, van mejorando, se van transformando. Se observa como a medida que el tiempo pasa, uno de los aspectos más importantes en la formación de toda persona es su educación. La educación entonces queda también inscrita en ese proceso de cambio, el cual va de la mano con el desarrollo tecnológico que la ciencia nos brinda, es por eso que la presente investigación se abordará lo que se percibe en relación con la evolución que ha tenido la educación.

\section{La calidad en la formación virtual}

Una formación virtual de calidad debe responder a unos requerimientos técnicos y metodológicos que satisfagan las necesidades de los usuarios, es decir que proporcione la posibilidad de consultar materiales didácticos de calidad materiales que le aporten conocimientos y no solo información y medios estables para la comunicación entre docente y estudiante dentro del ámbito educativo.

La docencia a nivel superior va desarrollando alternativas para que la enseñanza se vaya transformando, por los nuevos tiempos, exigencias y educación actualizada. Las nuevas exigencias en la educación se centran en la mejora del proceso educativo, y en ese sentido la integración de las Tics facilita aspectos relacionados con la mejora del trabajo individual, la autonomía del alumnado, la facilidad para el desarrollo del trabajo en equipo u colaborativo, la posibilidad de modificar y adaptar los métodos de evaluación y la interacción bidireccional entre el docente y el estudiante.

La incorporación de las tecnologías de la información y la comunicación en educación superior supone una apuesta por una universidad realmente competitiva que trata de 
potenciar el aprendizaje permanente, lo que va incidir poderosamente en el fortalecimiento de la calidad y en la expansión de las universidades a distancia.

Realmente con la ayuda de las Tics se obtiene una educación competitiva basada en la calidad del buen uso de los docentes y de los estudiantes, marcando una progresión sistemática con resultados muy ambicioso que justamente evoluciona positivamente en los procesos en la enseñanza - aprendizaje.

La educación virtual se centra en el ámbito educativo facilitando la interactividad y conectividad entre estudiantes y docentes, compañeros de clase y grupos de trabajo. Es una estrategia educativa que permite la aplicación de nuevos métodos enfocados al desarrollo del aprendizaje, los cuales están centrados en el estudiante y en la participación activa.

\section{Metodología}

El método científico fue el proceso a través de la observación de fenómenos sociales, como es la manera que interactúan docentes y alumnos a través de las aulas virtuales la forma como se realizó esta investigación, cuyo objetivo es establecer la percepción que tienen los docentes sobre el uso de las plataformas virtuales en la Universidad de Cotopaxi, extensión la Mana, donde se tomó un universo de 80 docentes de las distintas áreas, posteriormente se analizaron los datos para determinar las conclusiones. El método de investigación es el explicativo al establecer relaciones entre distintos aspectos de la realidad. El método utilizado es la observación, tomando la información como se presentó en el campus. En cuanto al alcance la investigación es teórica, sustentando así el tema de estudio en el marco teórico y desarrolla conclusiones mediante el hallazgo. La metodología aplicada es la documental sustentado la investigación en información previa, combinada con la investigación de campo, apoyando la información documental con datos que provienen, de entrevistas, y observaciones.

\section{Resultados}

A pesar de las múltiples ventajas que ofrece el recurso virtual no se pueden desconocer los riesgos potenciales por el mal uso que se le puede dar, a través de su uso. Mediante el modelo educativo el docente diseña la estrategia didáctica que va transmitir al estudiante, el mismo que le permite su concentración y participación activa dentro del aula de clases y así construir nuevos conocimientos para un mejor aprendizaje.

El plan de estudios centra su atención en el desempeño de los estudiantes a través del uso de nuevos conocimientos, actitudes y habilidades ante la actividad o solución de un problema.

Tabla 1. Ventajas y desventajas de la enseñanza virtual en la educación superior

\begin{tabular}{|l|l|}
\hline \multicolumn{1}{|c|}{ VENTAJAS } & \multicolumn{1}{|c|}{ DESVENTAJAS } \\
\hline $\begin{array}{l}\text { Se siente personalizado en el trato con el docente y } \\
\text { sus compañeros }\end{array}$ & $\begin{array}{l}\text { Inasistencia de estructura pedagógica en la } \\
\text { información y multimedia. }\end{array}$ \\
\hline $\begin{array}{l}\text { Puede adaptar el estudio a su horario personal, } \\
\text { teniendo acceso a la enseñanza, no viéndose } \\
\text { perjudicados aquellos que no pueden acudir }\end{array}$ & $\begin{array}{l}\text { Dificultades organizativas, problemas técnicos y } \\
\text { altos costos de mantenimiento. }\end{array}$ \\
\hline
\end{tabular}




\begin{tabular}{|c|c|}
\hline $\begin{array}{l}\text { periódicamente a clase por motivos de trabajo o } \\
\text { distancia, etc. }\end{array}$ & \\
\hline $\begin{array}{l}\text { Puede realizar sus participaciones de forma } \\
\text { meditada gracias a la posibilidad de trabajar off- } \\
\text { line. }\end{array}$ & $\begin{array}{l}\text { Temor a que los estudiantes vean los medios con } \\
\text { la pasividad con que se mira un programa de } \\
\text { televisión, caracterizado por una tendencia al } \\
\text { facilismo inmediato, inconveniente para } \\
\text { aprender ciertos contenidos. }\end{array}$ \\
\hline $\begin{array}{l}\text { Podrá seguir el ritmo de trabajo marcado por el } \\
\text { docente y por sus compañeros de curso. }\end{array}$ & $\begin{array}{l}\text { La tendencia a trabajar cualquier aspecto o } \\
\text { contenido de forma virtual, dejando de lado el } \\
\text { uso de medios más sencillos como } \\
\text { retroproyector. }\end{array}$ \\
\hline $\begin{array}{l}\text { El estudiante tiene un papel activo, que no se limita } \\
\text { recibir información, sino que forma parte de su } \\
\text { propia formación. }\end{array}$ & $\begin{array}{l}\text { Falta de una estructura pedagógica adecuada y } \\
\text { diseñada en los procesos cognitivos y las formas } \\
\text { de aprender de los estudiantes. }\end{array}$ \\
\hline $\begin{array}{l}\text { Existe el feed-back de información, de manera que } \\
\text { el docente conoce si el estudiante responsable al } \\
\text { método y alcanza los objetivos fijados } \\
\text { inicialmente. }\end{array}$ & $\begin{array}{l}\text { Una mala conexión de internet, falencias en el } \\
\text { equipo o material de trabajo pueden generar } \\
\text { retrasos e interrupciones. }\end{array}$ \\
\hline $\begin{array}{l}\text { Se beneficia de las ventajas de los distintos } \\
\text { métodos de enseñanza y medios didácticos } \\
\text { tradicionales, evitando los inconvenientes de los } \\
\text { mismos. }\end{array}$ & $\begin{array}{l}\text { Sin una rutina de estudios programada, se puede } \\
\text { descontrolar los horarios para otras actividades. }\end{array}$ \\
\hline
\end{tabular}

\section{Fuente: Autores}

El estudiante de la educación virtual aprende de forma más activa pues no sólo recibe la instrucción del docente, sino que aprende a través de la búsqueda de información, la autorreflexión y las diversas actividades que realiza de manera individual y colaborativa.

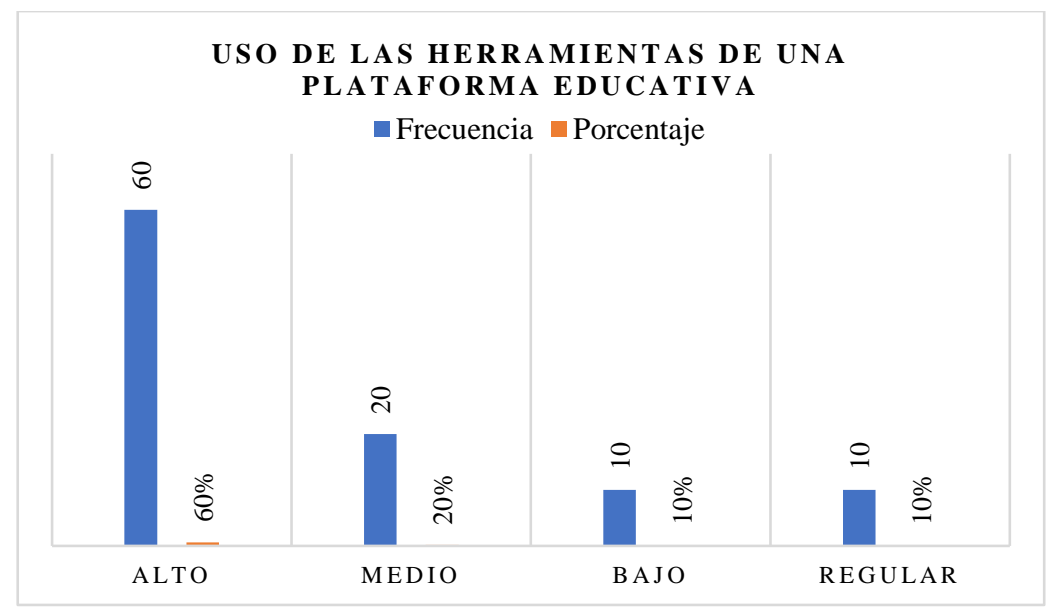

Figura 1. Uso de las aulas virtuales

\section{Elaborado por: Autores}

El 60\% de los docentes están capacitados en el uso de una plataforma educativa, el $20 \%$ de ellos están familiarizados con las herramientas, el $10 \%$ se encuentran en un nivel básico y el restante $10 \%$ de los docentes tiene un conocimiento escaso de estas herramientas tecnológicas. Porcentualmente el $80 \%$ del total de docentes usan adecuadamente una aula virtual, lo que ayuda significativamente a la capacitación del alumnado. 


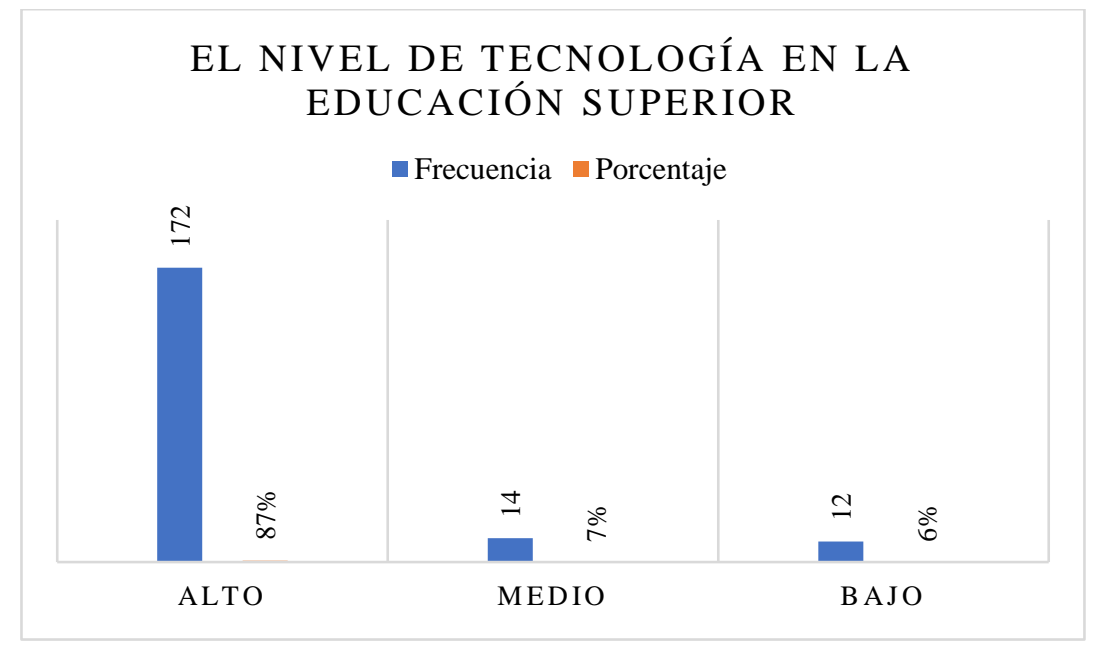

Figura 2. Tecnología en la educación superior

Elaborado por: Autores

En lo que se refiere a la percepción que tienes los docentes del nivel de tecnologías, un $87 \%$ considera que tiene un nivel alto, el $7 \%$ nivel medio y el $6 \%$ nivel bajo. Lo cual muestra que si existen los medios tecnológicos necesarios para impartir una educación de calidad permitiendo enfrentar los desafíos del proceso enseñanza - aprendizaje dentro de las aulas de clases.

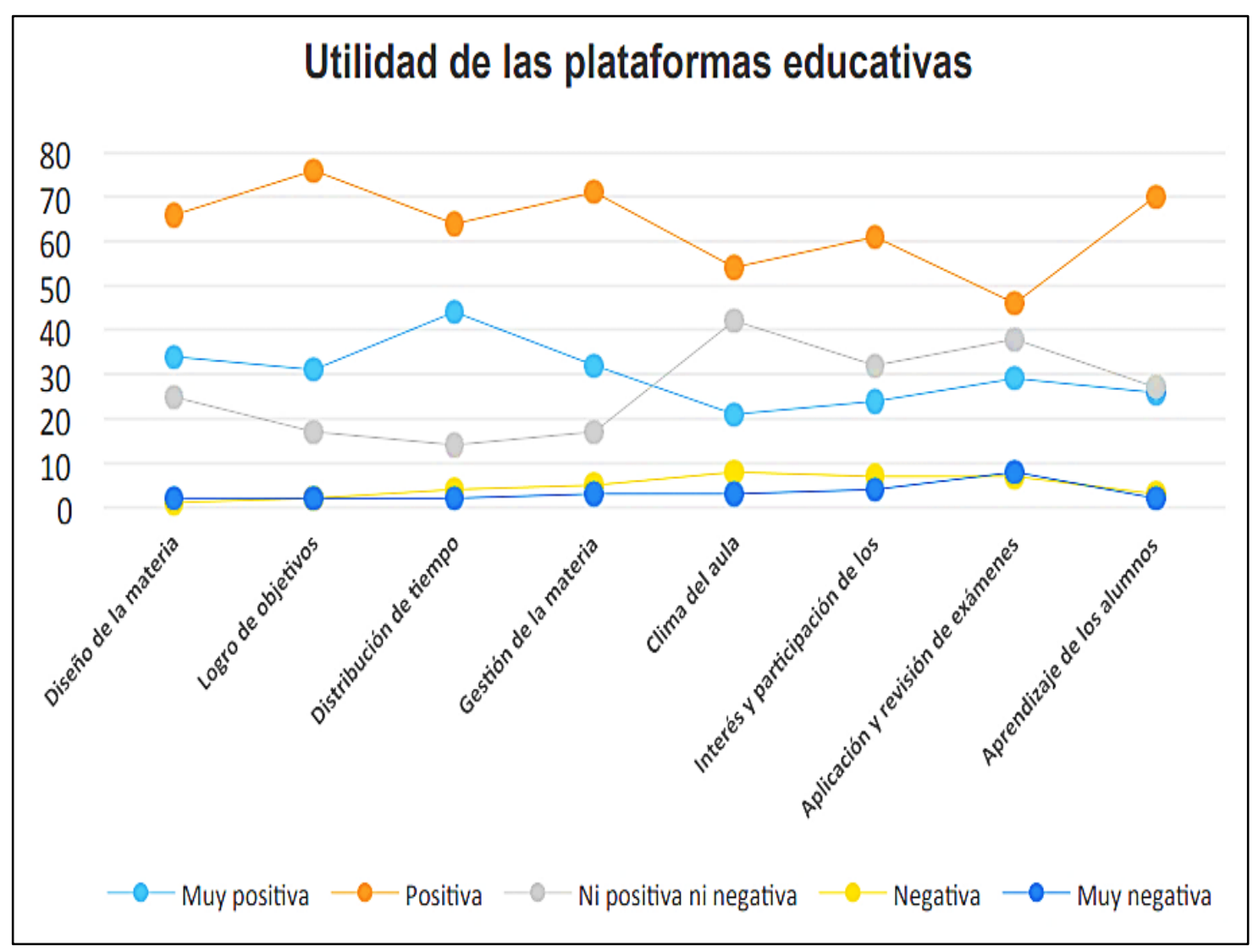

Figura 3. Utilidad de las plataformas educativas

Elaborado por: Autores 
Los docentes consideran que la utilidad de la plataforma en los ocho aspectos manejados en la encuesta es positiva o muy positiva. Sobresale el aprendizaje de los alumnos con el $75 \%$. Todo esto apoya los resultados realizados a través de la investigación sobre el análisis del impacto académico con el uso de una plataforma virtual en la Universidad de Cotopaxi, extensión la Mana. El 92\% de los profesores consideraron que la utilización de la plataforma institucional ha facilitado el contacto con los alumnos, mayor dedicación al preparar clase, genera mayor actitud positiva en el desarrollo académico.

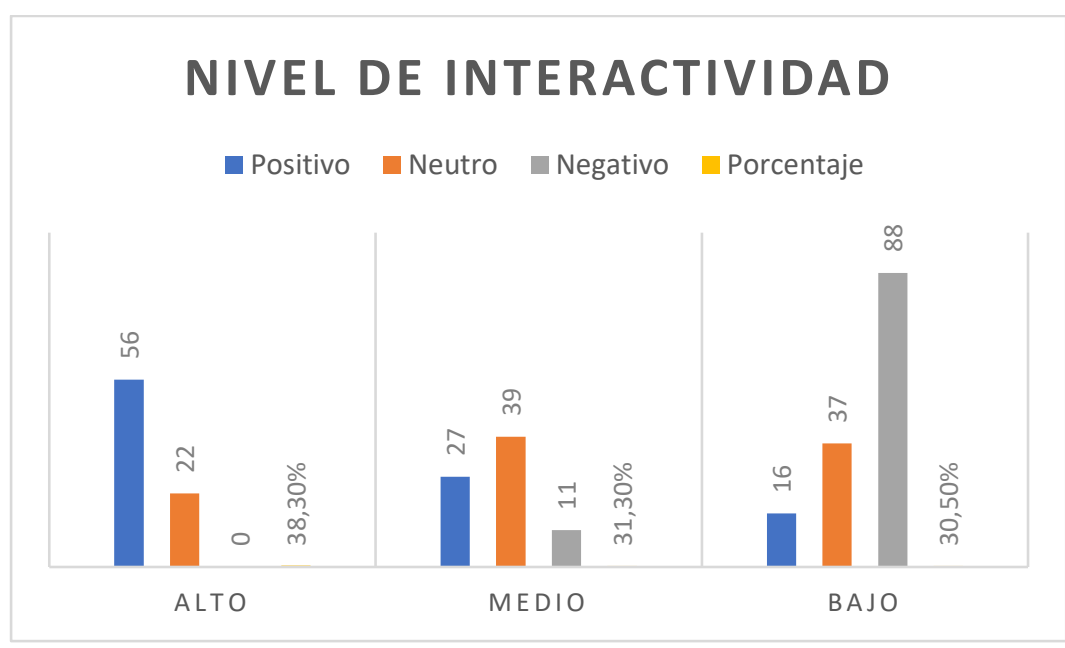

Figura 4. Nivel de interactividad

Elaborado por: Autores

Se obtuvo que los docentes perciben como herramientas principales y de mayor uso a la distribución de material, encargar actividades individuales, mandar y recibir mensajes, encargar actividades en equipo y retroalimentar y responder dudas; el nivel de uso de las herramientas tecnológicas es alto con un porcentaje de $38.3 \%$, el nivel medio es de $31.3 \%$ y bajo es de $30.5 \%$.

\section{Conclusiones}

$\checkmark$ Los docentes consideran que en la educación virtual el aprendizaje está centrado en el alumno y su participación activa en la construcción de conocimientos le asegura un aprendizaje significado.

$\checkmark$ Las plataformas virtuales usadas en la educación superior, juega un papel relevante en la renovación pedagógica mediante el proceso de enseñanza aprendizaje entre docente y estudiante.

$\checkmark$ Los docentes establecen la importancia de las plataformas virtuales o entornos de aprendizaje como medios para crear, administrar y gestionar de manera más flexible los contenidos académicos a través del internet.

\section{Referencias Bibliográficas}

Abrigo, I. (2015) Aula Virtual una herramienta para dinamizar el proceso de enseñanzaaprendizaje. Riobamba Ecuador. Pág. 12. ISBN: 978-84-608-3627-8. 
Bernad, E., \& Camacho, M. (2016). La Aula Virtual contenidos y elementos. España: Mc-Graw-Hill Interamericana de España S.L. Págs. 1000. ISBN: 8448612612.

Chamba, L. (2015) Escenarios de Aprendizaje para la inclusión y cohesión social. Universidad de Loja. Ecuador. EDUTEC. Pág. 12. ISBN: 978-84-608-3627-8.

Delgado, M. (2010) Propuesta acerca de un Multimedia - Virtual. Venezuela. Editorial Universitaria. Pág. 6. ISBN: 978-959-16-1305-9.

Domínguez, J. (2013) La Educación a distancia. Chimbote, Perú. Primera Edición. Págs. 352. ISBN: 978-612-46446-0-3

Gallo, R. (2013) Aulas virtuales su integración a la educación presencial. Sembrando experiencias. Uruguay Educa. Pág. 11.

Gómez, L. (2012) Importancia de los programas Virtuales en la Educación Superior. Investigación Educativa. Vol. $15 \mathrm{~N}^{\circ} 27,113-126$. ISSN: 1728-5852.

Khvilon, E. (2002) Aprendizaje abierto y a distancia. Montevideo, Uruguay. Edición TRILCE. Págs. 11. ISBN: 9974-32-348-7.

Lepez, L. (2014) La intervención educativa en línea, un dispositivo pedagógico en la formación docente. Buenos Aires, Argentina. Ciencia, Tecnología, Innovación y Educación. Págs. 12. ISBN: 978-84-7666-210-6.

Machado, A. (2004) Educar con Aulas Virtuales. Orientaciones para la innovación en el proceso de enseñanza y aprendizaje. Edición: Primera. Págs. 197. ISBN: 84-7774$147-6$.

Moreno, R. (2014) La Aulas Virtuales en el proceso de enseñanza aprendizaje. Revista Pedagógica Maestro y Sociedad. Santiago Cuba. Vol. 11. N³. Pág. 7. ISSN: 1815-4867.

Porter, L. (1997) Creating the Virtual Classroom: Distance Learning with the Internet. New York, USA. ISBN: 0471178306

Sierra, C. (2012) Educación Virtual aprendizaje autónomo y construcción de conocimiento. Bogotá. Editorial Politécnico Grancolombiano. Págs. 103. ISBN: 978-958-8721-16-3.

Vargas, R. (2004) Perspectivas docentes en la Educación Superior. Transformando la enseñanza en las instituciones de educación superior de la retórica a la realidad virtual. Revista Interinstitucional de Investigación Educativa. México. Vol. 5 $\mathrm{N}^{\circ} 009$. ISSN: 1665-0824.

Zambrano, W. (2012) Modelo de aprendizaje Virtual para la Educación Superior. ECO Ediciones. Bogotá. ISBN: 978-958-648-763-4.

\section{LCiencia}




\section{Para citar el artículo indexado.}

Velasteguí López, P., Chamba Melo, S., Quille Chilborazo, W., \& Velastegui López, E. (2019). Plataformas virtuales y su impacto en la Educación Superior. Explorador Digital, 3(4), 66-78. https://doi.org/10.33262/exploradordigital.v3i4.916

\section{\Ciencia}

El artículo que se publica es de exclusiva responsabilidad de los autores y no necesariamente reflejan el pensamiento de la Revista Explorador Digital.

El articulo queda en propiedad de la revista y, por tanto, su publicación parcial y/o total en otro medio tiene que ser autorizado por el director o editor de la Revista Explorador Digital.

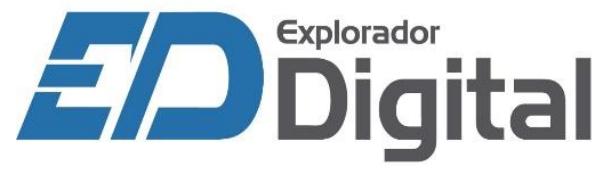

